\title{
Effects of acute treatment with statins on cerebral autoregulation in patients after aneurysmal subarachnoid hemorrhage
}

\author{
Ming-Yuan Tseng, M.D., M.Phil., M.Sc., Marek Czosnyka, Ph.D., \\ Hugh Richards, Ph.D., John D. Pickard, F.R.C.S., M.Chir., F.Med.Sci., \\ aNd Peter J. KirkPatrick, F.R.C.S.(SN)
}

Department of Neurosurgery, Addenbrooke's Hospital, University of Cambridge, United Kingdom

\begin{abstract}
Object. The authors previously have demonstrated that acute treatment with pravastatin after aneurysmal subarachnoid hemorrhage (SAH) can ameliorate vasospasm-related delayed ischemic neurological deficits (DINDs). In the current study, they test the hypothesis that these effects are associated with improvement in indices describing autoregulation of cerebral blood flow.

Methods. In this double-blind study, 80 patients between the ages of 18 and 84 years who had aneurysmal SAH were randomized equally to receive either $40 \mathrm{mg}$ of oral pravastatin or placebo once daily for up to 14 days (medication was started $1.8 \pm 1.3$ days after ictus). Autoregulation was measured using a daily transient hyperemic response test (THRT) on transcranial Doppler ultrasonography (800 measurements in 80 patients), and data were compared between the pravastatin and placebo groups and between patients with or without vasospasm, DINDs, or unfavorable outcome. Measurement of autoregulation also was performed using the pressure-reactivity index, a moving correlation coefficient between mean arterial and intracranial pressures (Days 0-5, 132 measurements in 32 patients).

There was no difference in baseline autoregulation indices between the trial groups. The members of the pravastatin group not only had a shorter duration of impaired autoregulation but also had stronger transient hyperemic response ratios (THRRs) bilaterally. A negative correlation existed between the mean flow velocity in the middle cerebral artery and THRRs. Onset of DINDs occurred when bilateral autoregulation failed. On Days 3, 4, and 5, the pressure-reactivity index correlated significantly with ipsilateral impaired autoregulation.

Conclusions. The neuroprotective effects of acute treatment with pravastatin following aneurysmal SAH are associated with enhancement of autoregulation. A routine and daily assessment of cerebral autoregulation by using the THRT may help identify patients at high risk of DINDs.
\end{abstract}

\section{KEY WORDS • cerebral ischemia • subarachnoid hemorrhage • vasospasm • statin • autoregulation}

A NEURYSMAL SAH has an incidence rate of 8 cases per 100,000 persons per year in the UK. Although this accounts for only $5 \%$ of all strokes in the UK, aneurysmal SAH is of significant clinical and socioeconomic importance because it particularly affects those who are relatively young (median age 52 years) and thus has a disproportionate effect on the loss of productive life. The early mortality rate reaches $20 \%$, and only $47 \%$ of patients are discharged directly home after successful interventions, with the rest needing further care in the referring hospital or a rehabilitation unit. The overall unfavorable outcome is as high as $38 \%$, which is associated with older patients as well as those with poor clinical grades and high Fisher grades on CT scans, ${ }^{7}$ larger aneurysms or aneurysms located on the posterior circulation, and comorbidi-

Abbreviations used in this paper: $\mathrm{CI}=$ confidence interval; $\mathrm{CT}=$ computed tomography; DINDs = delayed ischemic neurological deficits; $\mathrm{ICP}=$ intracranial pressure $\mathrm{MCA}=$ middle cerebral artery; $\mathrm{SAH}=$ subarachnoid hemorrhage; $\mathrm{TCD}=$ transcranial Doppler; THRR $=$ transient hyperemic response ratio; THRT $=$ THR test; $\mathrm{UK}=$ United Kingdom. ties (for example, hypertension or ischemic heart disease). Within the UK, this means that there are approximately 3500 patients with aneurysmal SAH either dying or sustaining severe disabilities every year. ${ }^{12}$

Cerebral autoregulation is an intrinsic self-defense mechanism against secondary ischemia that maintains a stable blood flow during fluctuations of the cerebral perfusion pressure. ${ }^{5}$ Impaired autoregulation has been known to correlate with poor outcome after head injuries. ${ }^{3}$ Indeed, daily assessment of autoregulation helps identify patients and situations associated with high risk of secondary ischemia. ${ }^{16}$

Autoregulation can be measured conveniently at the bedside by using the THRT on TCD ultrasonography. ${ }^{9}$ When the THRT is used for day-to-day assessments after aneurysm clip placement, there is a relationship with the clinical outcome. ${ }^{11}$ Another noninvasive measurement of autoregulation is the pressure-reactivity index, which is a moving correlation coefficient between the ICP and the mean arterial pressure. ${ }^{3}$ A pressure-reactivity index value greater than 0.2 has been found to reflect poor cere- 
brovascular reactivity and a value less than -0.2 demonstrates excellent reactivity in patients with head injuries. However, whether the pressure-reactivity index can be applied in cases of aneurysmal SAH is unclear.

We have previously demonstrated that acute treatment with pravastatin ameliorates cerebral vasospasm and reduces the incidence of related DINDs and death in patients following aneurysmal SAH. ${ }^{19}$ In this study, we assess the hypothesis that these effects were associated with improved autoregulation as defined using the THRT and pressure-reactivity index, and we explore the predictive value and mutual association between these indices.

\section{Clinical Material and Methods}

\section{Patient Population}

Data relating to clinical course, bedside monitoring, and TCD indices were extracted from the pravastatin trial. ${ }^{19}$ In this double-blind study, 80 patients between the ages of 18 and 84 years who had an aneurysmal SAH and who were admitted to the Department of Neurosurgery, Addenbrooke's Hospital were randomized to receive either $40 \mathrm{mg}$ of pravastatin sodium (Lipostat; Bristol-Myers Squibb Pharmaceuticals, Ltd., Hounslow, Middlesex, UK) or placebo (lactose) once daily. Trial medications were begun within 72 hours of ictus and continued for up to 14 days. Baseline data included age, sex, medical history, and initial aneurysmal SAH grade according to the World Federation of Neurosurgical Societies. ${ }^{6}$ Radiological information included the Fisher grade on CT scans, presence of hydrocephalus and/or intraventricular hemorrhage, and aneurysm location on cerebral angiography. Exclusion criteria were nonaneurysmal SAH, pregnancy, preictal statin therapy, and contraindications to statin use (for example, history of liver or renal dysfunction, or alanine aminotransferase level > 50 U/L). After admission, the clinical management of the condition in each patient followed department standards, including the administration of nimodipine and moderate intravenous fluid supplement. When there were clinical indications for continuous ICP monitoring, patients were admitted to the neurosciences critical care unit and an ICP probe (Codman MicroSensor; Johnson \& Johnson Medical, Ltd., Berkshire, UK) or an external ventricular drain was inserted into the nondominant frontal region. For symptomatic vasospasm salvage, "triple-H" therapy was administered. ${ }^{13}$ None of the patients underwent endovascular angioplasty (balloon angioplasty or endovascular vasodilator therapy). Factors that might affect the outcome were documented, including ventriculitis, sepsis, mode of aneurysm treatment (endovascular compared with clip placement), and immediate postoperative deficits.

\section{Examinations Using TCD Ultrasonography}

We adopted daily TCD ultrasonography (DWL MultiDop X4; DWL Elektronische Systeme GmbH, Singen, Germany) performed using a 2-MHz probe mounted on a specially designed head frame as a surrogate method to measure cerebral vasospasm. Systolic, diastolic, and mean flow velocities in both MCAs were captured (50- to 60$\mathrm{mm}$ depths) and recorded using in-house designed BioSan software. ${ }^{16}$ Vasospasm and severe vasospasm were defin- ed as demonstrating values of greater than 1.2 and $2 \mathrm{~m} / \mathrm{sec}-$ ond, respectively, with the Lindegaard ratio for both greater than 3, which has high predictive values for the presence of significant vasospasm on cerebral angiography. ${ }^{21}$

\section{Examinations for Cerebral Autoregulation}

Detailed methods for the assessment of cerebral autoregulation by using the THRT and pressure-reactivity index have been described in previous publications. ${ }^{9,16}$ In the current study, two carotid compressions lasting $5 \mathrm{sec}-$ onds with a 2-minute interval were performed after a minimum of a 10-second baseline data recording. The criteria for an acceptable THRT score included a sudden decrease in MCA flow velocity at the onset of compression, a stable TCD signal during compression, and a minimum of $30 \%$ decrease in flow velocity with no blood pressure instability. The THRR is calculated as follows: THRR = $\mathrm{FV}_{\text {systolic }}$ (hyperemia)/ $\mathrm{FV}_{\text {systolic }}$ (baseline), where $\mathrm{FV}$ means flow velocity. As shown in Fig. 1, autoregulation was defined as normal (THRR $\geq 1.10$ ) or impaired (THRR $<$ 1.10). ${ }^{16}$ The pressure-reactivity index was calculated as a moving correlation coefficient between 40 consecutive samples of mean values for ICP and arterial blood pressure averaged for a period of 6 seconds. ${ }^{3}$ The data were averaged for the whole period of recordings obtained from Days 0 to 5 .

\section{Definition of DIND and Outcome}

Vasospasm-related DIND was defined as development of focal neurological deficits and/or a drop in the Glasgow Coma Scale score by 2 points or more ${ }^{14}$ and was associated with severe vasospasm on TCD ultrasonography. Other conditions that could have caused neurological deterioration in the study patients (for example, hydrocephalus, intracerebral hemorrhage, surgical complications, metabolic abnormalities, infection) were excluded using repeated imaging (CT, xenon $\mathrm{CT}$, and/or cerebral angiography) and metabolic screening. Immediate postoperative deficits were defined as new neurological deficits that were evident on recovery from general anesthesia. Patient disability at discharge was recorded according to the modified Rankin Scale as favorable (score of 1 or 2) or unfavorable (score of 3-6). ${ }^{1}$

\section{Statistical Analysis}

All analyses were performed on an intention-to-treat basis, and probability values were two-sided. Data were presented as the mean and with 95\% CIs. Analysis was performed using statistical software (STATA Intercooled 8.0 for Windows; STATA Corp., College Station, TX). Analysis was performed separately on the side ipsilateral and the one contralateral to the ruptured aneurysm. The THRR and the maximum mean flow velocity of the MCA were averaged every 3 days from Days 0 to 14. Daily pressure-reactivity index results from each patient were averaged to produce a patient-oriented database to satisfy the independence assumption for linear regression. Data were compared between the pravastatin and placebo groups and between patients with or without vasospasm, DINDs, or unfavorable outcome on the t-test. Probability values less than 0.05 were considered significant. 


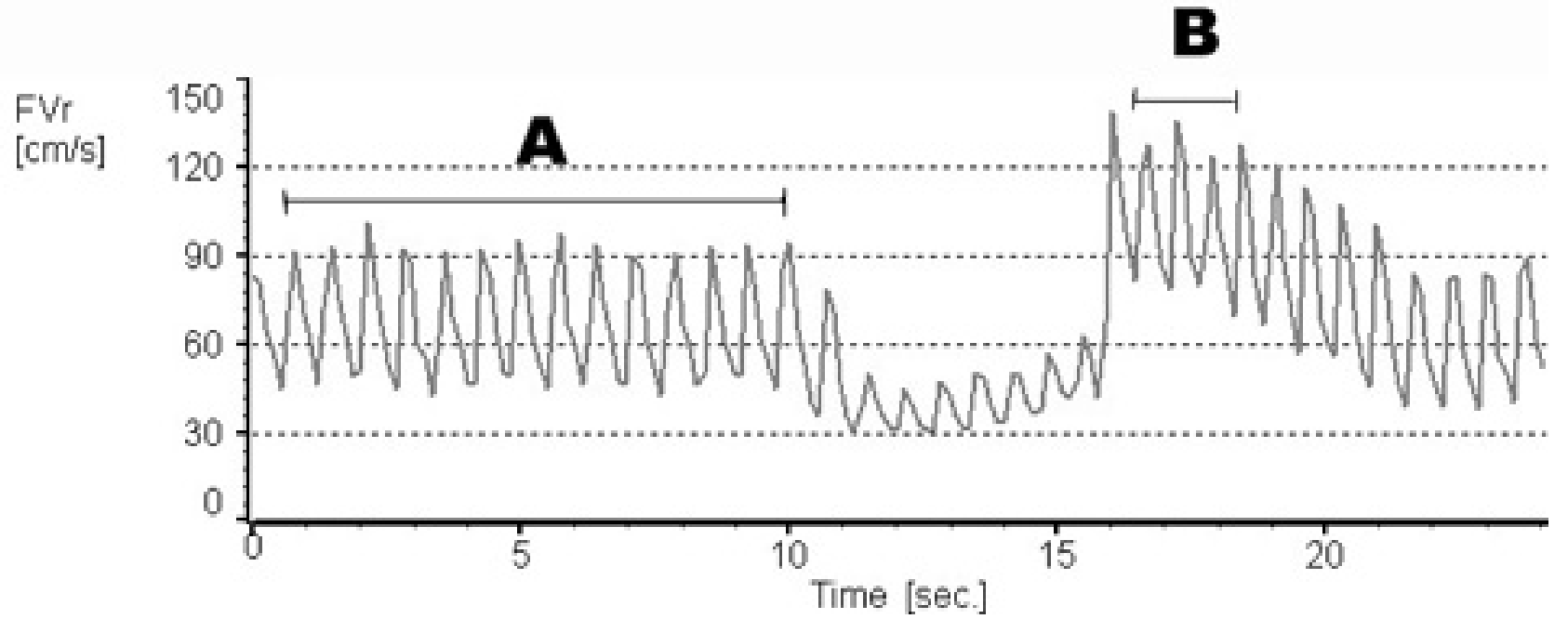

FIG. 1. Graph of a flow velocity waveform of the MCA during a THRT. A: Baseline reading. B: Hyperemic response. The response is normal (THRT ratio 1.25$) . \mathrm{FVr}=$ flow velocity right side.

\section{Results}

\section{Patient Data}

Trial medication was begun within 1.8 days $(95 \% \mathrm{CI}$ $0-4.3$ days) after the ictus. Forty-two $(52.5 \%)$ of the 80 patients demonstrated vasospasm on TCD ultrasonography, including $25(62.5 \%)$ of the 40 patients who received placebo and $17(42.5 \%)$ of the 40 who received pravastatin. Nineteen $(23.8 \%)$ of the 80 patients had severe vasospasm, including $12(30 \%)$ of the 40 who received placebo and seven (17.5\%) of the 40 who received pravastatin. Vasospasm-related DINDs occurred in $14(17.5 \%)$ of the 80 patients (12 [30\%] of the 40 patients who received placebo and two [5\%] of the 40 patients who received pravastatin) and were associated with new cerebral infarcts on CT scans. Daily THRRs were available for all but one patient, in whom the image quality was unsatisfactory for interpretation because of a thicker temporal bone on the contralateral side. All 80 patients were included for the final analysis of THRR compared with vasospasm incidence, DINDs, and effects of statins. Thirty-one of the 32 patients with ICP monitoring or external ventricular drainage had suitable data for pressure-reactivity index assessment. These patients were examined during the acute postictus period when intensive care was required. Therefore only $15 \%$ of the THRT data overlapped with data from the pressure-reactivity index examinations (132 of nearly 800).

\section{The TCD-Derived Autoregulation Index and Statin Treatment}

On Day 0, no difference in the TCD-derived autoregulation indices was found between the two groups. Patients in the pravastatin group had a shortened duration of autoregulation impairment on both sides (ipsilateral side: 3.0 days, 95\% CI 1.9-4.1 days; contralateral side: 1.6 days, 95\% CI 0.9-2.3 days) compared with those in the placebo group (ipsilateral side: 5.3 days, 95\% CI 3.9-6.8 days; contralateral side: 3.7 days, 95\% CI 2.3-5.1 days). The mean number of days by which the impairment was short- ened was 2.4 days (95\% CI 3.2-5.1 days, $\mathrm{p}=0.011$ ) for the ipsilateral side and 2.08 days (95\% CI 0.6-3.6 days, $\mathrm{p}=$
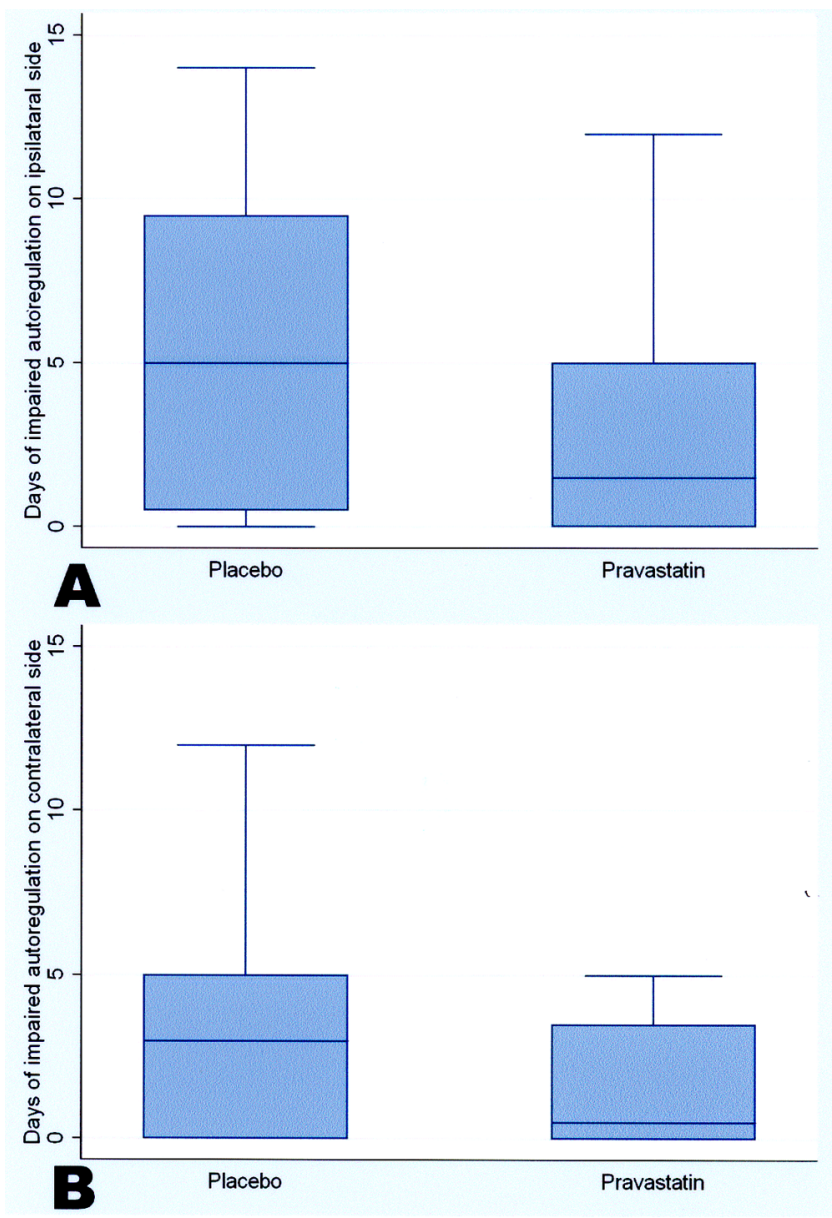

FIG. 2. Box plots of impaired autoregulation in the ipsilateral (A) and the contralateral (B) sides of the ruptured aneurysm. According to the t-test, the probability value was 0.011 for the ipsilateral side and 0.008 for the contralateral side. 

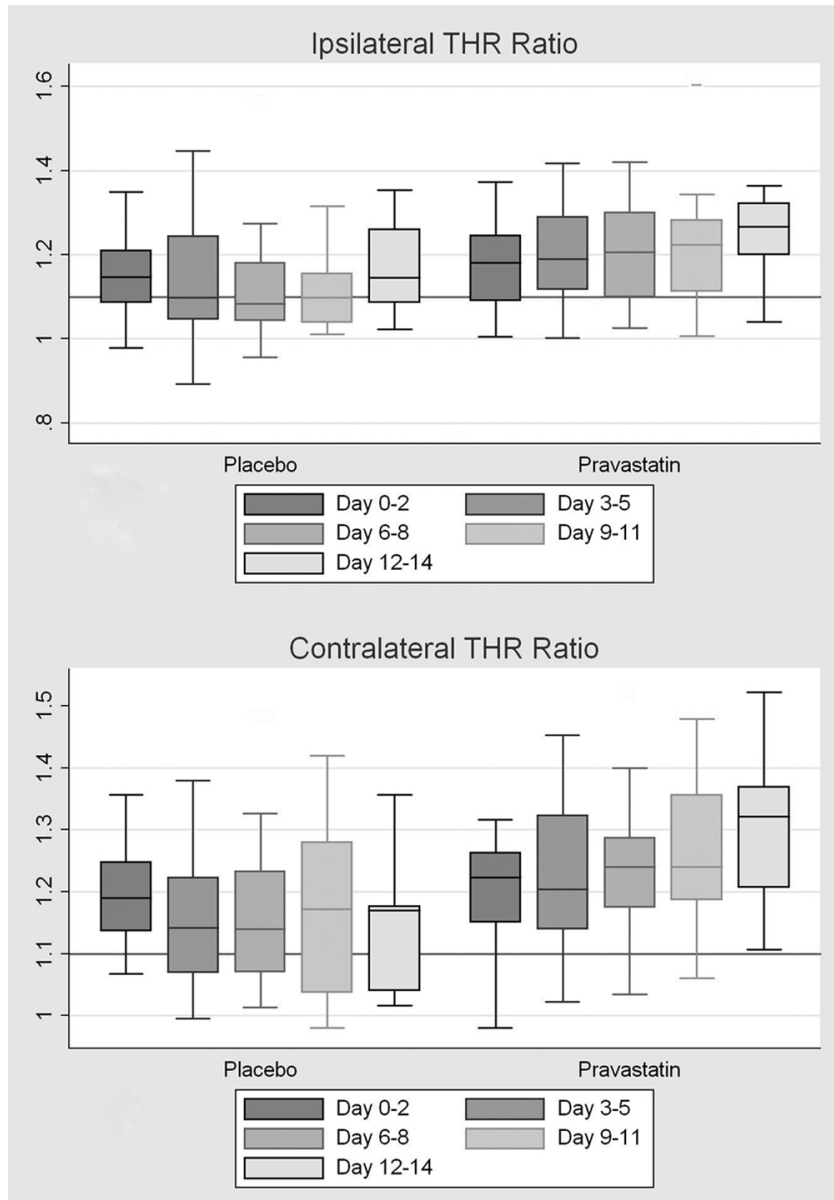

FIG. 3. Box plots comparing the THRRs of the pravastatin and placebo groups on the ipsilateral (upper) and contralateral (lower) sides. Each box represents the median value. Each whisker represents the 75th (upper hinge) and the 25th (lower hinge) percentiles. The horizontal line at the THRR of 1.1 indicates the break between the positive (above the line) or negative (below the line) cerebral autoregulation.

0.008) for the contralateral (Fig. 2). After Day 3, the THRR on both sides was consistently higher in the pravastatin group (Fig. 3). The maximum differences were $+9 \%(95 \% \mathrm{CI}+1.8$ to $+16.2 \%$, Days $9-11, \mathrm{p}=0.013)$ on the ipsilateral side and $+13.9 \%(95 \% \mathrm{CI}+5.2$ to $+12.3 \%$, Days $12-14, \mathrm{p}=0.002$ ) on the contralateral.

\section{Correlation Among Autoregulation Indices and Mean Blood Flow Velocity}

There was a negative correlation between the pressurereactivity index and the ipsilateral THRR from Day 3 to Day $5(\mathrm{r}=-0.635, \mathrm{p}=0.008)$; a similar correlation, but one of borderline significance, was found between the pressure-reactivity index and the contralateral THRR $(\mathrm{r}=$ $-0.748, p=0.064)$. There were significantly negative correlations between the THRR and the mean flow velocity of the ipsilateral MCA from Day 0 to Day $14(\mathrm{r}=-292.14$ to $-143.69, \mathrm{p}=0.002$ ), and similar correlations were found on the contralateral side after Day $3(\mathrm{r}=-204.54$ to $-77.64, \mathrm{p} \geq 0.05$ ) but not during the acute period (Days $0-3)$. Correlations between the pressure-reactivity index and mean flow velocity can be seen on both sides $(\mathrm{r} \sim 0.40$, $\mathrm{p}<0.02)$.

\section{Correlations Among Autoregulation Indices and Patients With and Without Vasospasm, DINDs, and Unfavorable Outcome}

No significant difference was found between the patients with good and poor grades in terms of their initial THRT responses, THRRs, pressure-reactivity index scores, and durations of impaired autoregulation. In patients who had severe vasospasm on the ipsilateral side, the corresponding THRR was significantly lower from Day 0 to Day 14, reaching the maximum level on Days 9 to 11 $(-10.8 \%, 95 \% \mathrm{CI}-4.2$ to $-18.3 \%, \mathrm{p}=0.002)$, and the THRR on the contralateral side was lower during the period of DIND (Days 9-11, $-11.2 \%, 95 \%$ CI -2.4 to $-20 \%$, $\mathrm{p}=0.016$, Fig. 4). Therefore, onsets of DINDs occurred around the time when the THRR on both sides significantly decreased (Days 9-11). Patients with vasospasm, severe vasospasm, DINDs, or unfavorable outcome had
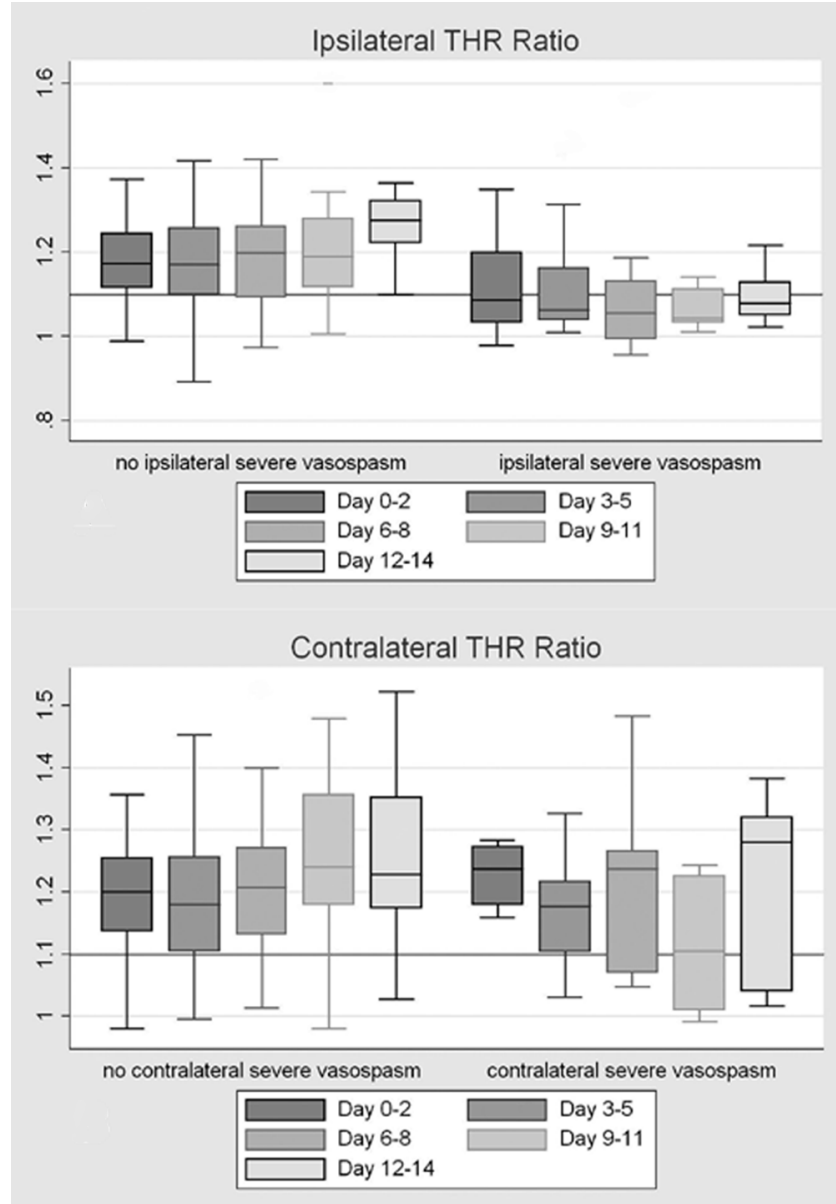

FIG. 4. Box plots comparing the THRRs of patients with and without severe vasospasm on the ipsilateral (upper) and contralateral (lower) sides. Each box represents the median value. Each whisker represents the 75th (upper hinge) and the 25th (lower hinge) percentiles. The horizontal line at the THRR of 1.1 indicates the break between the positive (above the line) or negative (below the line) cerebral autoregulation. 
TABLE 1

Comparison in durations of impaired autoregulation in patients with aneurysmal SAH

\begin{tabular}{|c|c|c|c|c|}
\hline \multirow[b]{2}{*}{ Side } & \multicolumn{2}{|c|}{$\begin{array}{l}\text { Days of Autoregulation } \\
\text { Impairment }(95 \% \mathrm{CI})^{*}\end{array}$} & \multirow[b]{2}{*}{ Difference $(95 \% \mathrm{CI})$} & \multirow[b]{2}{*}{$\mathrm{p}$ Value } \\
\hline & $\mathrm{w} /$ & w/o & & \\
\hline \multicolumn{5}{|l|}{ ipsilat } \\
\hline vasospasm & $6.4(5.0-7.8)$ & $2.1(1.2-3.0)$ & $4.3(2.7-5.9)$ & $<0.001$ \\
\hline severe vasospasm & $8.2(6.6-9.7)$ & $3.1(2.1-4.0)$ & $5.1(3.2-7.1)$ & $<0.001$ \\
\hline DIND & $8.6(6.8-10.4)$ & $3.2(2.3-4.1)$ & $5.4(3.2-7.5)$ & $<0.001$ \\
\hline unfavorable outcome & $5.2(3.8-6.7)$ & $3.2(2.0-4.3)$ & $2.1(0.3-3.9)$ & 0.02 \\
\hline \multicolumn{5}{|l|}{ contralat } \\
\hline vasospasm & $4.2(2.4-6.0)$ & $2.1(1.2-2.9)$ & $2.1(0.4$ to 3.8$)$ & 0.017 \\
\hline severe vasospasm & $4.6(2.0-7.3)$ & $2.4(1.6-3.3)$ & $2.2(-0.4$ to 4.8$)$ & 0.099 \\
\hline DIND & $5.0(2.2-7.8)$ & $2.2(1.4-2.9)$ & $2.8(0.9$ to 4.8$)$ & 0.006 \\
\hline unfavorable outcome & $3.0(1.7-4.2)$ & $2.4(1.4-3.4)$ & $0.5(-2.1$ to 1.0$)$ & 0.497 \\
\hline
\end{tabular}

* Assessed using TCD ultrasonography.

significantly longer durations of impaired autoregulation as measured by the THRT, particularly on the ipsilateral side, compared with those without such events (Table 1).

\section{Discussion}

Effective compensatory mechanisms of cerebral blood flow are important for avoiding cerebral ischemia during vasospasm..$^{14,15}$ For patients in this study who experienced DINDs, autoregulation in both the ipsilateral and contralateral sides failed simultaneously. In this situation, acute resuscitation by induced hypertension and intravascular volume enhancement may lead to rapid development of cerebral edema. ${ }^{5}$ Thus, effective therapy for preventing or treating vasospasm-related DINDs should be oriented to improving autoregulation.

Why is autoregulation lost after aneurysmal SAH-induced vasospasm? The early onset of impaired autoregulation seen in poor-grade patients is closely associated with severe vasospasm and cerebral infarcts, ${ }^{18}$ implying that the load of subarachnoid blood is the cause of these complications. The negative correlation between the THRR and the mean flow velocity of the MCA indicates that high-speed blood flow causes adverse effects on cerebral autoregulation. Excessive flow velocities during vasospasm have been known to produce greater shear force on the vascular wall, potentially interfering with endothelial function..$^{10}$ Therefore, there seems to be a cycle of adverse events following endothelial damage, including autoregulation impairment, vasospasm, and eventual cerebral ischemia. $^{20}$

The direct contact between oxyhemoglobin from subarachnoid blood and cerebrovascular adventitia also may be important because it results in denervation and loss of neurogenic control of cerebral arteries, a depletion of endothelium-derived nitric oxide, and an increased production of endothelin. ${ }^{17}$ Impaired activation of the potassium channels in vascular smooth muscle and enhanced platelet aggregation and leukocyte adhesion are also implicated. ${ }^{17}$

In this 14-day trial, we found that pravastatin therapy can effectively ameliorate vasospasm, including its incidence, duration, and severity. Conversely, patients in whom pravastatin was discontinued seemed to start to suffer vasospasm-related DINDs. ${ }^{19}$ These findings indicate that these vascular effects are prompt and relatively shortlived. ${ }^{2,8}$ Therefore, future trials of statins to treat patients with aneurysmal SAH may have to adopt courses of 3 or more weeks.

We did not find predictive functions for the pressurereactivity index in terms of clinical outcome and adverse events among patients with aneurysmal SAH that were similar to those seen in patients with head injuries. ${ }^{4}$ This is probably because of the relatively small sample of 32 patients or the shorter duration of measurement (Days 0-5), which failed to correlate with data on vasospasm. Another possibility is that the ICP-derived property of the pressure-reactivity index cannot detect efficiently the side difference of vasospasm-related complications. Therefore, the value of the pressure-reactivity index measurement in patients with aneurysmal SAH will need to be assessed in a larger group of patients or a study of longer durations of ICP measurements, principally for those patients presenting in coma, for whom ICP monitoring is more routine.

\section{Conclusions}

In this study, we have shown that the neuroprotective effects of acute treatment with pravastatin following aneurysmal SAH are associated with the enhancement of normal autoregulation. A routine and daily assessment of cerebral autoregulation by using THRT may help identify patients at high risk of DINDs. An assessment in which the pressure-reactivity index is used in the acute phase after aneurysmal SAH is less helpful in this respect.

\section{Disclaimer}

None of the authors has a financial interest in the devices used in this study.

\section{References}

1. Bamford JM, Sandercock PA, Warlow CP, Slattery J: Interobserver agreement for the assessment of handicap in stroke patients. Stroke 20:828, 1989

2. Chen J, Zhang ZG, Li Y, Wang Y, Wang L, Jiang H, et al: Statins induce angiogenesis, neurogenesis, and synaptogenesis after stroke. Ann Neurol 53:743-751, 2003

3. Czosnyka M, Smielewski P, Kirkpatrick P, Laing RJ, Menon D, 
Pickard JD: Continuous assessment of the cerebral vasomotor reactivity in head injury. Neurosurgery 41:11-17, 1997

4. Czosnyka M, Smielewski P, Kirkpatrick P, Piechnik S, Laing R, Pickard JD: Continuous monitoring of cerebrovascular pressure-reactivity in head injury. Acta Neurochir Suppl 71: 74-77, 1998

5. Czosnyka M, Smielewski P, Piechnik S, Steiner LA, Pickard JD: Cerebral autoregulation following head injury. J Neurosurg 95:756-763, 2001

6. Drake CG: Report of World Federation of Neurological Surgeons Committee on a universal subarachnoid hemorrhage grading scale. J Neurosurg 68:985-986, 1988 (Letter)

7. Fisher CM, Kistler JP, Davis JM: Relation of cerebral vasospasm to subarachnoid hemorrhage visualized by computerized tomographic scanning. Neurosurgery 6:1-9, 1980

8. Gertz K, Laufs U, Lindauer U, Nickenig G, Bohm M, Dirnagl $\mathrm{U}$, et al: Withdrawal of statin treatment abrogates stroke protection in mice. Stroke 34:551-557, 2003

9. Giller CA: A bedside test for cerebral autoregulation using transcranial Doppler ultrasound. Acta Neurochir (Wein) 108:7-14, 1991

10. Hatake K, Wakabayashi I, Kakishita E, Hishida S: Impairment of endothelium-dependent relaxation in human basilar artery after subarachnoid hemorrhage. Stroke 23:1111-1117, 1992

11. Lam JM, Smielewski P, Czosnyka M, Pickard JD, Kirkpatrick PJ: Predicting delayed ischemic deficits after aneurysmal subarachnoid hemorrhage using a transient hyperaemic response test of cerebral autoregulation. Neurosurgery 47:819-826, 2000

12. Lindsay KW, Langham J, Kirkpatrick PJ, Shaw MDM, Gholkar AR, Molyneux A, et al: National Study of Subarachnoid Haemorrhage: Final Report of an Audit Carried out in 34 Neurosurgical Units in the UK and Ireland Between 14 September 2001 to 13 September 2002. London: The Royal College of Surgeons of England, 2006

13. Origitano TC, Wascher TM, Reichman OH, Anderson DE: Sustained increased cerebral blood flow with prophylactic hypertensive hypervolemic hemodilution ("triple-H" therapy) after subarachnoid hemorrhage. Neurosurgery 27:729-740, 1990

14. Pickard JD, Murray GD, Illingworth R, Shaw MD, Teasdale GM, Foy PM, et al: Effect of oral nimodipine on cerebral in- farction and outcome after subarachnoid hemorrhage: British aneurysm nimodipine trial. Br Med J 298:636-642, 1989

15. Ratsep T, Asser T: Cerebral hemodynamic impairment after aneurysmal subarachnoid hemorrhage as evaluated using transcranial Doppler ultrasonography: relationship to delayed cerebral ischemia and clinical outcome. J Neurosurg 95:393-401, 2001

16. Smielewski P, Czosnyka M, Kirkpatrick P, Pickard JD: Evaluation of the transient hyperemic response test in head-injured patients. J Neurosurg 86:773-778, 1997

17. Sobey CG, Quan L: Impaired cerebral dilatation responses to NO and PDE V inhibition after subarachnoid hemorrhage. Am J Physiol 277:H1718-H1724, 1999

18. Soehle M, Czosnyka M, Pickard JD, Kirkpatrick PJ: Continuous assessment of cerebral autoregulation in subarachnoid hemorrhage. Anesth Analg 98:1133-1139, 2004

19. Tseng MY, Czosnyka M, Richards H, Pickard JD, Kirkpatrick PJ: Effects of acute treatment with pravastatin on cerebral vasospasm, autoregulation, and delayed ischemic deficits after aneurysmal subarachnoid hemorrhage: a phase II randomized placebo-controlled trial. Stroke 36:1627-1632, 2005

20. Voldby B, Enevoldsen EM, Jensen FT: Cerebrovascular reactivity in patients with ruptured intracranial aneurysms. J Neurosurg 62:59-67, 1985

21. Vora YY, Suarez-Almazor M, Steinke DE, Martin ML, Findlay JM: Role of transcranial Doppler monitoring in the diagnosis of cerebral vasospasm after subarachnoid hemorrhage. Neurosurgery 44:1237-1248, 1999

Dr. Ming-Yuan Tseng was supported by the Raymond and Beverly Sackler Studentship, University of Cambridge. This project was sponsored by the Addenbrooke's Hospital National Heath Services Trust and the University of Cambridge Dr. Marek Czosnyka is on unpaid leave from Warsaw University of Technology, Poland.

Manuscript received July 10, 2006.

Accepted in final form August 18, 2006.

Address reprint requests to: Ming-Yuan Tseng, M.D, M.Phil, M.Sc, Box 167, Department of Neurosurgery, Addenbrooke's Hospital, Cambridge CB2 2QQ, United Kingdom. email: mty22@ cam.ac.uk. 